\title{
Photosensitizers for Photogalvanic Cells in the Chemistry Classroom
}

\author{
Claudia Bohrmann-Linde*, Diana Zeller \\ Department of Chemistry Education, University of Tübingen, Auf der Morgenstelle 18, 72076 Tübingen, Germany \\ *Corresponding author: claudia.bohrmann-linde@uni-tuebingen.de
}

\begin{abstract}
Alternative solar cells on the basis of titanium dioxide are subject of intense research. For the integration of this topic into the chemistry curriculum a set of experiments with photogalvanic cells has been developed. Titania phototelectrodes need to be irradiated with UV light, but the spectrum of solar light contains only little UV radiation. Thus photosensitizers are needed that harvest visible light. Here we present a series of experiments with different dye solutions that can serve as an alternative to the priorly used anthocyanins in the chemistry classroom. The dyes presented are more stable and also work as sensitizers in titania-based photogalvanic cells.
\end{abstract}

Keywords: titanium dioxide, titania, photogalvanic cells, photosensitization, light, natural dyes, semiconductor, solar cells, hands-on chemistry-lab, sustainable development education

Cite This Article: Claudia Bohrmann-Linde, and Diana Zeller, "Photosensitizers for Photogalvanic Cells in the Chemistry Classroom.” World Journal of Chemical Education vol. 6, no. 1 (2018): 36-42. doi: 10.12691/wjce-6-1-7.

\section{Introduction and Background}

Photogalvanic cells have been under investigation for the integration into the chemistry classroom for some years already [1]. Photogalvanic cells are cells similar to galvanic cells such as the Daniell cell, which is one of the standard experiments carried out in chemistry classrooms worldwide. In contrast to galvanic cells, photogalvanic cells contain a semiconductor-based photoelectrode and deliver a photovoltage or a photocurrent upon irradiation. The photosensitive part is a thin layer of titanium dioxide nanoparticles, $\mathrm{TiO}_{2}$, on a conductive glass plate (TEC glass). Nanoscale titanium dioxide is used, which forms a mesoporous network with a great surface area. This titania photoelectrode is also used in dye sensitized solar cells (DSSCs, also known as Grätzel cells) that have been subject of intense research ever since O'Reagan and Grätzel proposed the first prototype in Nature more than 25 years ago [2]. Dye sensitized solar cells have also previously been proposed for the chemistry classroom [3] [4]. As the aspect of sensitization is also a key issue in photosynthesis, the experiments described in this paper may well be integrated in an interdisciplinary project.

It is our claim as representatives of curricular innovation research to develop experiments and concepts starting from established topics and moving onwards to innovative aspects from research and technology. Therefore our proposed didactic concept starts in the context of electrochemistry and energy conversion and storage with photogalvanic cells in a 2-pot-version, similar to the set-up of galvanic cells, and moves on to more flat and almost dry cells, c.f. Figure 1 [5] that can also be constructed as transparent photogalvanic cells. A teaching unit on photogalvanic cells opens the perspective of dealing with solar cells using a chemical approach in the chemistry classroom. The advantage is that one can not only work with ready-made products but construct light-driven cells in a hands-on lab activity. Doing so, the students are introduced to current academic research in the field of alternative solar cells, in this case dye-sensitized titania-based solar cells. Teachers can use the teaching unit on (sensitized) photogalvanic cells as another building block in Education for Sustainable Development (ESD).

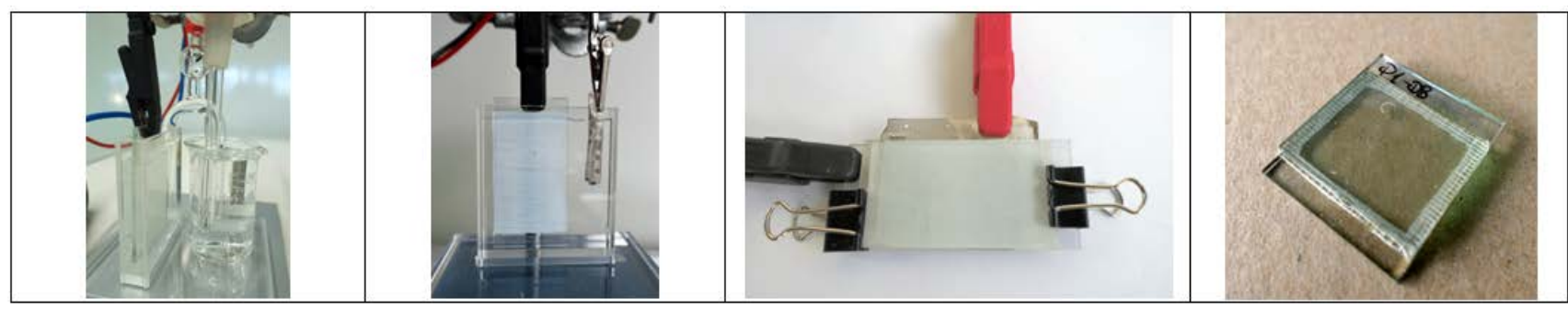

Figure 1. Different unsensitized photogalvanic cells using a titania-coated photoelectrode; from left to right: 2-pot-cell, 1-pot-cell, compact cell, transparent cell 
In the photogalvanic cells we first use a "white" untreated $\mathrm{TiO}_{2}$-photoelectrode that needs to be irradiated with light of wavelengths below 388nm, as the bandgap in titania (in the anatase modification) is $3,2 \mathrm{eV}$. So light sources that emit UV light or violet light are suitable. Irradiation of the titania-anode causes an electron to be promoted into the conduction band, leaving an electron deficit, an associated "hole", in the valence band (i). Thus the first step is the formation of electron-hole pairs in the semiconductor.

$$
\text { (i) }\left(\mathrm{TiO}_{2}\right)(\mathrm{s})+\mathrm{h} \cdot \mathrm{v} \rightarrow\left(\mathrm{TiO}_{2}\right)^{+}(\mathrm{s})+\mathrm{e}^{-}
$$$$
\text { ("hole") }
$$

If the electron deficit in the valence band is compensated by the oxidation of an electron donor (ii), the electron in the conduction band can move through the semiconductor towards the back contact and out into the external circuit.

(ii) $\left(\mathrm{TiO}_{2}\right)^{+}(\mathrm{s})+$ donor $\rightarrow\left(\mathrm{TiO}_{2}\right)(\mathrm{s})+$ donor $_{\mathrm{ox}}$

As the electrons move towards a counter electrode electrical energy is generated. Many of these processes then generate a measurable photocurrent. So the two essential steps are the formation and the separation of electron-hole-pairs, just as in other solar cells as well.

\section{The Concept of Photosensitization}

The greatest part of the spectrum of solar light is not UV-light. Thus the photoelectrode has to be modified with the aim of utilizing a wider range of the spectrum of visible light. This can be achieved by using photosensitizers that absorb light of the visible part of the spectrum of electromagnetic radiation. Photosensitization leads to a narrowing of the bandgap. It is a phenomenon that also takes place in plants. Chlorophyll dyes in the thylakoid membranes of green leaves absorb light from the visible part of the spectrum. Light absorption is followed by a cascade of steps in the leaves, comprising also several electron transfers. In sum, photons are converted into chemical energy which is stored in the plants.

In sensitized titania photoelectrodes the sensitizing dye is anchored to the semiconductor surface via chemical bonds. When dye-sensitized photoelectrodes are irradiated with light from the visible part of the spectrum of electromagnetic radiation, photons are absorbed by the dye-molecules. Upon irradiation an electron is promoted from the HOMO to the LUMO, the molecule is in its electronically excited state, c.f. (i) and step 1 in Figure 2. The electron is then transferred to the conduction band of titania, which is represented by $\mathrm{TiO}_{2} \bullet \mathrm{e}^{-}$in (ii) and by step $2 \mathrm{~b}$ in Figure 2. It finally moves out into the external circuit, represented by step 3 in Figure 2. In order to restore the dye, the electron deficit has to be compensated via the oxidation of a donor from the electrolyte, c.f. (iii) and step 2a in Figure 2.

(i) dye $+\mathrm{h} \bullet v \rightarrow$ dye*

(ii) dye* $+\mathrm{TiO}_{2} \rightarrow$ dye $\mathrm{ox}+\mathrm{TiO}_{2} \bullet \mathrm{e}$

(iii) dye $\mathrm{ox}_{\mathrm{ox}}+$ donor $\rightarrow$ dye + donor $_{\mathrm{ox}}$

The transfer of an electron from a donor to the hole in the HOMO of the dye molecule is fundamental for the transfer of the excited electron to the conduction band of titania, as it impedes the competing process of recombination of excited electron and hole. In fact, it has to take place almost simultaneously, which is represented by steps 2a and 2b in Figure 2.

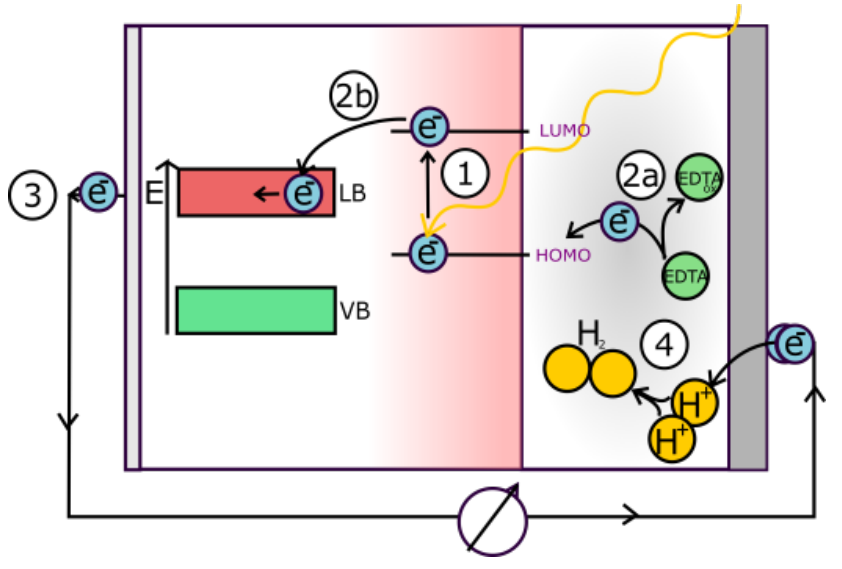

Figure 2. Processes in and at a sensitized photoelectrode

At the counter electrode, incoming electrons reduce protons from the solution, forming hydrogen molecules. Since the turnover is low, no hydrogen gas can be observed in the experiment within a regular lesson.

\section{Photosensitizers for Titania}

Photosensitizers that are suitable for the use in titaniabased cells have to meet with the following requirements:

- they should absorb in the visible region of the electromagnetic spectrum,

- they should be water-soluble or soluble in other solvents typically used in school chemistry,

- their molecules should contain functional groups that can chemically bind to titania and

- the position of the LUMO should be higher than that of the conduction band of the semiconductor, so electron transfer can take place (step $2 \mathrm{~b}$ in Figure 2).

In titania-based solar cells for technical applications classically organometallic complexes based on ruthenium [6] with relatively broad absorption spectra serve as sensitizing dyes, but there is a range of other dyes such as phorphyrin-derivatives $[7,8]$ under investigation. The stability of the dyes is a limiting factor in the use of DSSCs [9]. In order to avoid degradation caused by ambient humidity or oxygen the cells have to be sealed. Photodegradation is another limiting factor. For school purposes sealing is not necessary as titania-based photogalvanic cells or Grätzel cells are not in longterm use. Here the availability and price of the cell components play an important role.

In the past, anthocyanins have been proposed as suitable sensitizers for school purposes. Anthocyanins are the natural dyes in petals and many fruits [10]. They are readily available throughout the year, e.g. as frozen fruits, easy to handle and generally effective to show the main principle. One drawback though, is their short-lived stability when irradiated with light, especially in the presence of titania. Depending on the light source, it comes to a visible 
degradation of the dye, yielding in lower voltages within a school lesson. In fact, titania is a photocatalyst and promotes the photodegradation of anthocyanins [11]. After a few irradiation cycles an anthocyanine-sensitized photoelectrode is obviously degraded, see Figure 3. The degradation of anthocyanine dyes is further enhanced by the EDTA electrolyte, in cells with the redox mediator $\mathrm{KI} / \mathrm{I}_{2}$, as used in Grätzel cells, degradation takes place slower.

So the question arose, which other dyes could be used as more stable sensitizers for titania in photogalvanic cells in the school context. The idea was to test dyes of colors other than red, so one could possibly widen the spectrum of sensitizing dyes, which might eventually lead to a "cocktail" of sensitizer dyes. Prerequisites were that the dyes should be lowcost, easily available for schools and known to students from their everyday lives. More chemistry-related criteria for suitable sensitizers are: the dye molecules should have a conjugated $\pi$-electron system and hydroxyl-groups as functional groups for binding to $\mathrm{TiO}_{2}$, and for water-solubility. Finally there should be no safety hazards.

With respect to availability and occurrence in the students' everyday lives we have tested a number of different food colorants as well as spices such as saffron and turmeric. Especially saffron proved to work as a suitable dye for the use as alternative sensitizer for titania. The dye that is mainly responsible for the yellow color of saffron is crocin (Figure 4). Crocin is a carotene derivative with disaccharide substituents, which cause the dye to be water-soluble. The following procedures focus on experiments with saffron and the pure substance crocin. The experimental section presents a sequence of lab experiments useful for investigations on the dyes with respect to the preparations of the solutions, the properties, stability and, finally, the use as sensitizer in photogalvanic cells.
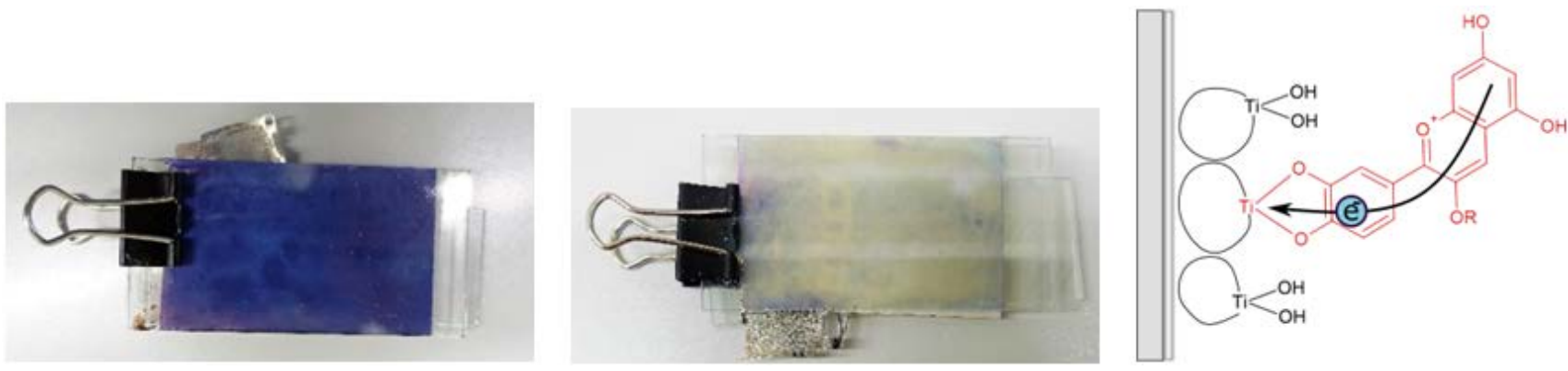

Figure 3. Anthocyanin-sensitized photoelectrodes a) before and b) after 15 minutes of irradiation with white light from a flashlight (X-Tactical 103) and c) anthocyanin molecule that is bound to a titania particle via the two vicinal OH-groups of the phenyl substituent
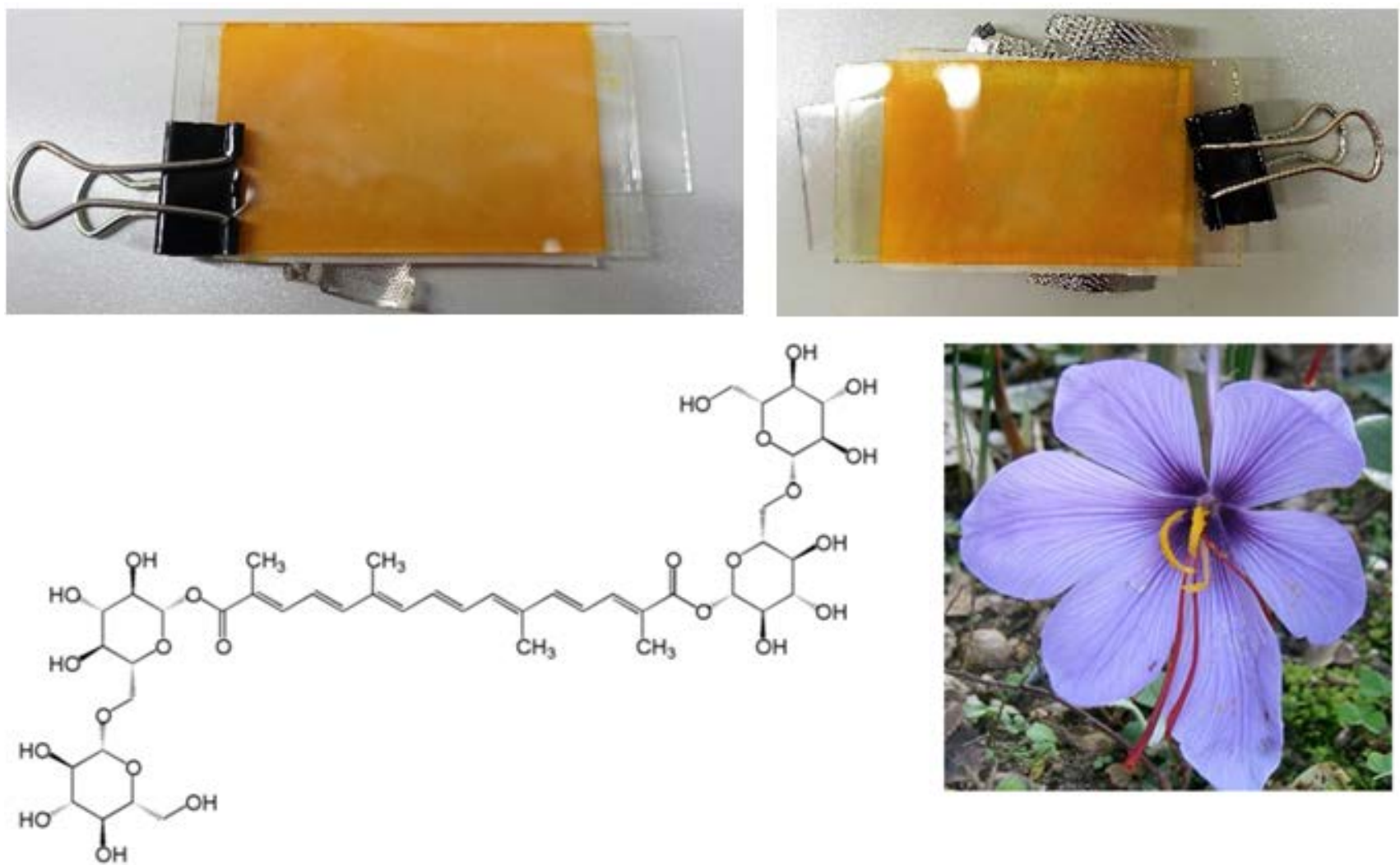

Figure 4. Crocin-sensitized photoelectrodes a) before and b) after 15 minutes of irradiation with white light from a flashlight (X-Tactical); c) structure of crocin; the dye is mainly responsible for the yellow color of saffron from the saffron crocus d) 


\section{Experimental Instructions}

\subsection{Hazards}

Currently the hazards of titania as cancerogen are discussed at EU level. Studies showed that the inhalation or the oral intake of titania nanoparticels can increase the risk of carcinogesis [12]. In our experiments students work with a suspension of nanoscale titania, so an inhalative exposition is not of relevance. In addition an adequate use of the substance will prevent an oral intake of titania nanoparticles in chemistry lessons.

While working with UV light, it is recommended to wear UV blocking glasses.

\subsection{Preparation of Titania Photoelectrodes and Sensitizing Solutions}

The photoelectrodes can be prepared by the doctor-blade method. A dilute acidic suspension of titania (P25, Evonik) is spread across the conductive side of a TEC glass and sintered as described on our homepage [13]. With this simple method students can prepare photoelectrodes within a regular school lesson and use it in the different kinds of photogalvanic cells. The resulting surfaces have an average thickness of below $20 \mu \mathrm{m}$ and a surface roughness of about $10 \mu \mathrm{m}$ as can be deduced from the height profile of 3D microscope images (Figure 5).

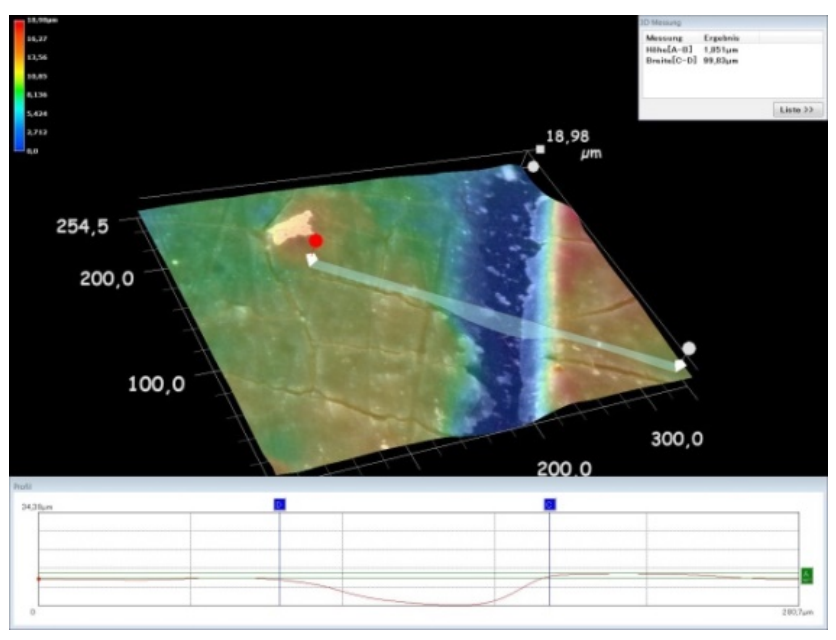

Figure 5. Surface of a titania photoelectrode that was scratched down to the conductive glass (blue areas). Measurements of the profile give first information about the thickness of the titania layer and surface roughness. (Keyence VHD-1000X)

Anthocyanins from raspberry juice are mainly used for dye sensitized solar cells in student experiments. The raspberry juice is prepared by mashing defrosted raspberries with distilled water in a ratio of three teaspoons of raspberries and $50 \mathrm{~mL}$ distilled water. After a few minutes the sediments will sink and the juice can be decanted.

It is recommended to deep freeze the solution in smaller portions that can be defrosted before experimenting. A drop of concentrated hydrochloric acid can be added to sterilize the dye.

For the crocin and saffron dyes $0,1 \mathrm{~g}$ of the substance are mixed with $40 \mathrm{~mL}$ distilled water. Crocin was obtained from Sigma Aldrich, at 15,46 €/g. The crocin solution has a greater concentration than the saffron dye, because the amount of the saffron was chosen to be affordable to schools. After use the solutions can be stored in an airtight container in the dark, so that they will last at least three months.

The sintered titania photoelectrode can be sensitized by dipping it into the respective dye solution for 5 minutes. After taking out the photoelectrode, it should be carefully rinsed with distilled water and gently dried with a blow dryer (cold air level).

\subsection{Absorption Spectra of Tested Dyes}

First of all absorption spectra of the three dye solutions (raspberry juice, crocin solution and saffron solution) were obtained by UV/VIS spectroscopy (Figure 6). The observed range of wavelengths of the near ultraviolet and visible spectrum was from 320nm to $800 \mathrm{~nm}$. The intensities of the absorbance vary due to different concentrations of dye solutions. As expected from the colors of the dye solutions the absorptions differ in their range of wavelength. In the visible part raspberry juice has its main absorption in a range from $470 \mathrm{~nm}$ to $570 \mathrm{~nm}$. So raspberry juice absorbs green light best. The crocin and saffron dyes have the same absorption spectrum, although the spectrum of crocin has a higher intensity. The main absorption in a range from 370nm to 520nm, therefore crocin and saffron solution mainly absorb blue light. Thus the natural product saffron and the pure substance crocin are an interesting alternative to raspberry juice.

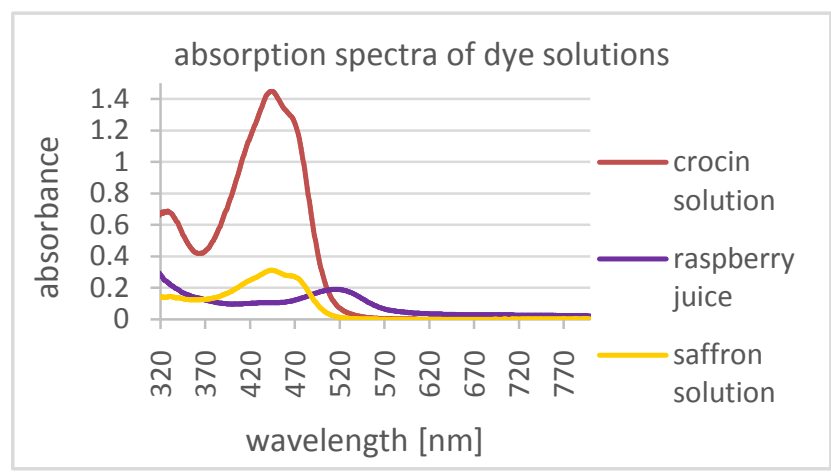

Figure 6. Absorption spectra of raspberry juice, saffron solution and crocin solution

\subsection{Binding on Titania}

A hint of the binding of the dyes to the titania surface is that the titania layer remains colored after rinsing with distilled water. The dyes seem to cover the whole surface. REM pictures proved not suitable for demonstrating bonds between the dye molecules and the titania particles. In order to get an impression of the surfaces of the sensitized titania photoelectrodes, light microscope images were taken with a magnification of 1,000×. (Keyence VHD-1000X).

The images in Figure 7 show that in each case the dyes almost evenly cover the titania surface. There are very few white and yellow spots. The height profile suggests that these are larger agglomerates of titania, very few of which are not covered by the dye. The cracks in the layer result from the cooling process which is relatively fast in order to prepare photoelectrodes within a chemistry lesson. The cracks have no significant influence on the cell performance. 

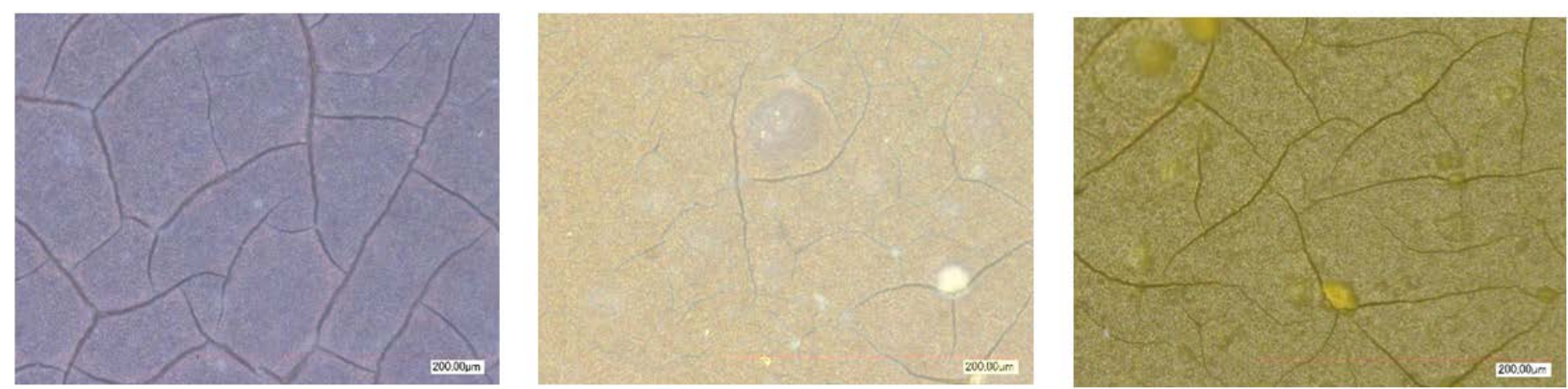

Figure 7. Light microscope images (1,000x) of titania surfaces, sensitized with a) raspberry juice, b) saffron solution, and c) with crocin solution (Keyence VHD-1000X)

\subsection{Photostability of the Dyes on the Sensitized Photoelectrode}

In the presence of titania the photosensitizers tend to degrade when irradiated with light. To compare the photostability of the sensitizer dyes the following simple experiment can be carried out from one lesson to the next. For each dye, two photoelectrodes are sensitized with a raspberry, saffron or crocin solution. One of each dye-sensitized photoelectrodes is placed on the window sill in the sunlight. A second sensitized photoelectrode is stored in a dark place.
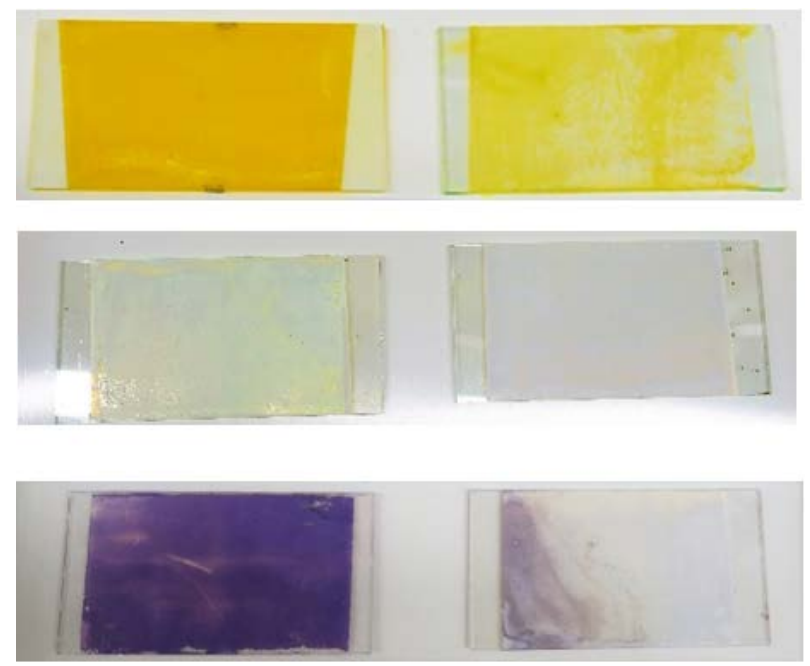

Figure 8. Bleaching of sensitizing dyes on titania (from top to bottom: crocin, saffron, anthocyanins)

Figure 8 shows significant differences between the photoelectrodes that were placed in the dark and the ones that were placed in sunlight after 24 hours. As it is difficult to determine the concentration of the dyes in the solutions of the natural products raspberry and saffron, the observed results allow only qualitative conclusions.

All sensitizers bleached visibly. Despite the differences in the dye concentrations of the sensitizer solutions one can deduce that crocin as a photosensitizer is more stable than anthocyanins from raspberry juice. Both dyes lead to an intensive coloring of the photoelectrode at the beginning, but the anthocyanin dyes almost faded completely after 24 hours. So the crocin dye may be regarded as a more stable sensitizer for titania in the school context.

In order to examine the influence of titania, this experiment can be repeated using filter paper soaked in a) an aqueous dye solution and b) in a suspension of titania and dye solution. The papers should then be cut in half und be stored in the dark and in the sunlight, respectively [11].

\subsection{Testing Alternative Sensitizers in a Photogalvanic Cell}

In order to test, if the crocin dye works as a sensitizer for titania, a one-pot-cell with a crocin-sensitized photoelectrode is set up and irradiated with different light sources. LED-based flashlights have proved very practical for use in the classroom. As UV light source a UV-LED flashlight (SDO-UV365 5W flashlight, Sidiou Group) with $\lambda=365 \mathrm{~nm}$ was used. For colored light a multicolor LED-flashlight (X-Tactical 103, LiteXpress) with red, green, blue and white light was used.

For a comparison and in order to show that the white, untreated titania photoelectrode is sensitive to UV-light but insensitive to less energetic colored light, a one-potcell was set up with a white photoelectrode as a reference beforehand (Figure 9b).

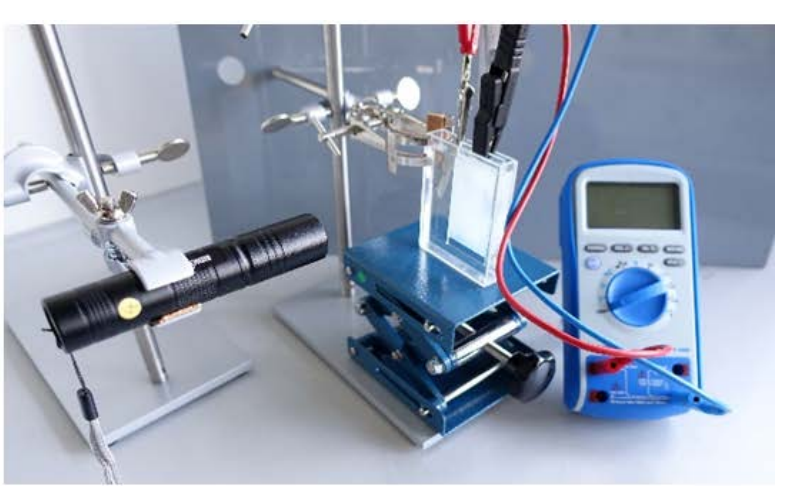

Figure 9. a) Photovoltages obtained in a 1-pot-cell with an untreated photoelectrode. Irradiation with UV light, white light, blue light and red light. b) Set-up of the experiment 
It is obvious that with the white photoelectrode irradiation with UV light leads to the generation of high photovoltages up to $700 \mathrm{mV}$ (Figure 9a). As soon as the irradiation pauses, the photovoltage drops. It rises again, if the irradiation continues. In contrast, the irradiation with white, blue and red light gives little to no measurable photovoltage. The observation that blue and white light lead to low photovoltages of about $50 \mathrm{mV}$ can be explained by the fact, that the titania used (P25, Evonik) is not pure anatase, but a mixture of anatase and some amounts of rutile. The band gap of rutile is $3,0 \mathrm{eV}$, so light of wavelengths below 413nm can form electron-hole-pairs in the rutile. To sum up, the white titania photoelectrode is quite insensitive to light of the visible spectrum.

In a second step a white photoelectrode is sensitized with a crocin solution as described in 3.2, placed into the one-pot-cell and irradiated in intervals by blue, green and red light from the X-Tactical flashlight. photovoltage of crocin sensitized photoelectrodes under the irridation of different light sources

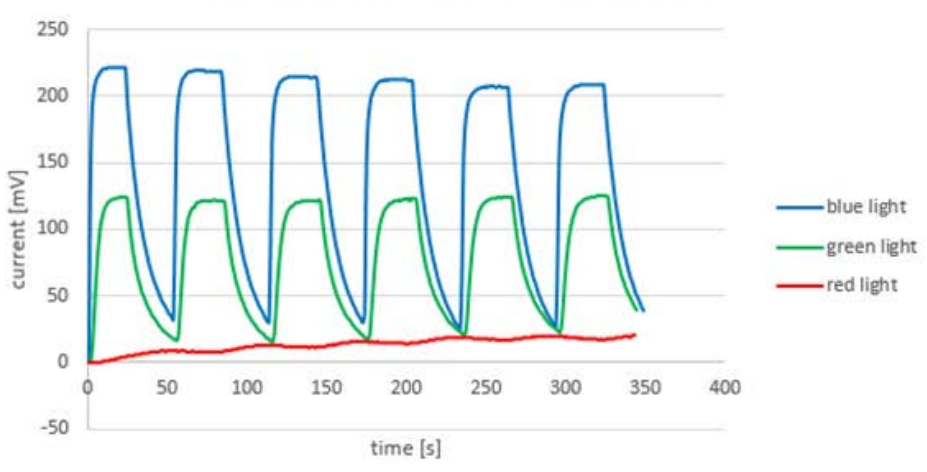

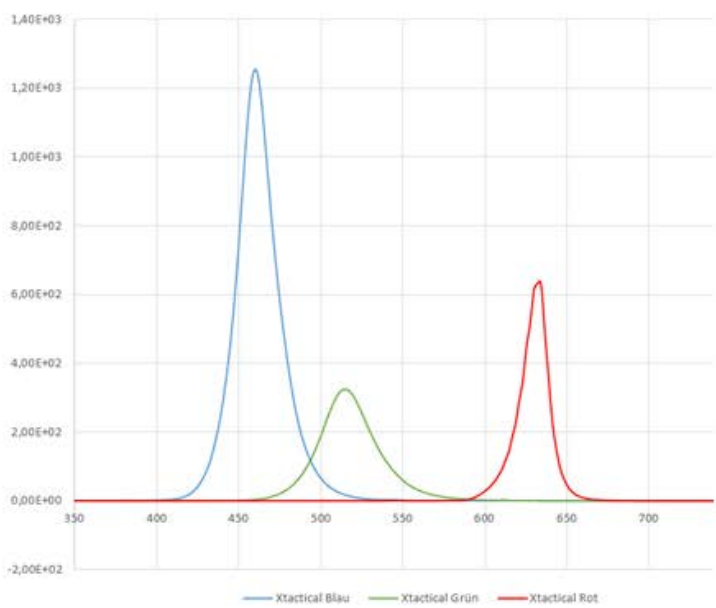

Figure 10. a) Photovoltages of crocin sensitized photoelectrodes in a 1-pot-cell, irradiated with different colors of light and b) emission spectra of the colored flashlight LEDs (X-Tactical)

The photoelectrode is photosensitive when irradiated by blue and green light. Irradiation with blue light yields the highest photovoltage of about $220 \mathrm{mV}$, which is more than a fourfold increase compared to the photovoltage of an unsensitized photoelectrode irradiated by blue light. This clearly indicates that crocin is an effective photosensitizer for titania.

The observation that blue light leads to higher photovoltages than green light can be correlated to the absorption spectrum of crocin solution (Figure 6 in 3.3), where the maximum absorption is in the region of 420-490 nm, thus in the region of blue light. This also matches the yellow color of the crocin solution. Red light is hardly absorbed by the crocin-sensitized photoelectrode and has not enough energy to surpass the bandgap of titania and/or the gap between HOMO and LUMO of the dye, leading to a negligible photovoltage.

Another aspect that could be considered is the intensity of the LEDs of the flashlight. Whereas the blue LEDs irradiate the sensitized photoelectrodes with the greatest intensity of all tested colored LEDs (Figure 10b), the intensitiy of the green LEDs is the lowest. The fact that the red light produces hardly any photovoltage, accounts for the hypothesis that it is mainly the absorption maximum that is the reason for higher voltages upon irradiation with blue light.

\section{Conclusion}

The topic DSSC solar cells as alternative to silicon-based solar cells has been intensively investigated in current scientific research. Our contribution serves as a bridge between academic research and dealing with this topic in the chemistry classroom. The experimental sequence on photogalvanic cells introduces the students to an alternative kind of solar cell which can be explored via a chemical approach. It can be integrated into the curriculum starting from the context of electrochemistry, if titania-based photoelectrodes are used in different kinds of photogalvanic cells. Different sensitizing dyes can be explored regarding their absorption characteristics, binding to titania, photodegradation and their use as sensitizers for titania photoelectrodes. This may also be realized in an interdisciplinary setting, e.g. in cooperation with biology (focus on photosynthesis) or physics (focus on siliconbased solar cells, photovoltaics, semiconductor devices). The sequence paves the way to dealing with solar cells with regard to teaching the use of renewable energies within Sustainable Development Education.

\section{References}

[1] C. Bohrmann-Linde, M.W. Tausch, Photogalvanic Cells for Classroom Investigations - A Contribution for the Ongoing Curriculum Modernization, J.Chem.Educ., 80 (2003), 1471-1473.

[2] B. O’Reagan, M. Grätzel, A low-cost, high-efficiency solar cell based on dye-sensitized colloidal $\mathrm{TiO}_{2}$ films, Nature 353 (1991), 737-740.

[3] G.P. Smestad, M. Grätzel, Demonstrating Electron Transfer and Nanotechnology: A Natural Dye-Sensitized Nanocrystalline Energy Converter; J.Chem.Educ., 75 (1998), 752-756.

[4] C. Bohrmann, M. Twellmann, M. W. Tausch, Vom galvanischen Element zur Solarzelle, NiU Chemie, 66 (2001), 12-16.

[5] C. Bohrmann-Linde, D. Zeller, Solarzellen ohne Silicium für den Chemieunterricht, Nachr. Chem., 65 (2017), 1236-1239.

[6] M. K. Nazeeruddin, A. Kay, I. Rodicio, R. Humphry-Baker, E. Mueller, P. Liska, N. Vlachopoulos, M. Grätzel, Conversion of light to electricity by cis-X2bis(2,2'-bipyridyl-4,4'dicarboxylate)ruthenium(II) charge-transfer sensitizers ( $\mathrm{X}=\mathrm{Cl}$-, $\mathrm{Br}-$, I-, CN-, and SCN-) on nanocrystalline titanium dioxide electrodes, J.Am. Chem. Soc., 115 (1993), 6382-6390. 
[7] S. Mathew, A. Yella, P. Gao, R. Humphry-Baker, B.F. Curchod, N. Ashari-Astani, I. Tavernelle, U. Rothlisberger, M.K. Nazeeruddin, M. Grätzel, Dye-sensitized solar cells with $13 \%$ efficiency achieved through the molecular engineering of porphyrin sensitizers, Nature Chem 6 (2014), 242-247.

[8] J.-J. Cid, J.-H. Yum, S.-R. Jang, M.K. Nazeeruddin, E. MartínezFerrero, E. Palomares, J. Ko, M. Grätzel, T. Torres, Phtalocyanines: Molecular Cosensitization for Efficient Panchromatic DyeSensitized Solar Cells, Angew. Chem. 119 (2007), 8510-8514.

[9] B. E. Hardin, H. J. Snaith, M. D. McGehee, The renaissance of dye-sensitized solar cells, Nature Photonics, 6 (2012), 162-169.
[10] C. Bohrmann-Linde, S. Krees, Anthocyane als Photosensibilisatoren für Titandioxid, PdN-ChiS, 54 (2005), 24-30.

[11] M. W. Tausch, C. Bohrmann-Linde, S. Krees, N. Meuter, J. Ibanez, I. Fernandez-Gallardo, I. Loblez-Leyzaola, G. AlessoRoblez, The Basis for Photocatalytic Writing, J.Chem.Educ., 88 (2011), 1116-1118.

[12] https://echa.europa.eu/de/-/titanium-dioxide-proposed-to-beclassified-as-suspected-of-causing-cancer-when-inhaled (12.12.17).

[13] http://www.uni-tuebingen.de/chemiedidaktik (12.12.17). 